\title{
CONTRIBUIÇÕES DA AULA DE GINÁSTICA ARTÍSTICA PARA O DESENVOLVIMENTO DAS HABILIDADES FUNDAMENTAIS
}

\author{
Suziane Peixoto Santos, Universidade Federal do Triângulo Mineiro - UFTM, Uberaba, \\ Minas Gerais - Brasil \\ Wagner Wey Moreira, Universidade Federal do Triângulo Mineiro - UFTM, Uberaba, \\ Minas Gerais - Brasil \\ Regina Simões, Universidade Federal do Triângulo Mineiro - UFTM, Uberaba, Minas \\ Gerais - Brasil \\ Aline Dessupoio Chaves, Universidade Federal do Triângulo Mineiro - UFTM, Uberaba, \\ Minas Gerais - Brasil \\ Michele Viviene Carbinatto, Universidade de São Paulo - USP, São Paulo, São Paulo - \\ Brasil
}

\section{RESUMO}

Este estudo investigou possíveis diferenças nos movimentos fundamentais entre préescolares que participam regularmente da prática de esportes e pré-escolares que participam apenas de Educação Física escolar, incluindo comparação entre as idades. Doze crianças que tiveram semanalmente duas aulas de EF formaram o grupo Educação Física (GEF) e outras doze que tiveram semanalmente duas aulas de EF e duas aulas de GA formaram o grupo GA. Para a avaliação dos padrões fundamentais de movimento, foi utilizado o Modelo de Avaliação Instrumental dos Movimentos Fundamentais. Os dados foram avaliados por componente conforme a lista de checagem, no qual cada padrão de movimento pode ser classificado em padrão inicial, elementar ou maduro. Os dados foram analisados em relação à distribuição (porcentagem) em cada nível de desenvolvimento. Os resultados indicaram que as crianças do GA apresentaram melhores resultados em relação ao GEF, exceto nos movimentos manipulativos. Desta forma, concluímos que as aulas de GA aliadas as de EF escolar contribuíram para um melhor desenvolvimento motor em relação às crianças que faziam apenas Educação Física escolar.

Palavras-Chave: Habilidades fundamentais; Ginástica artística; Educação Física escolar.

\section{CONTRIBUTIONS ARTISTIC GYMNASTICS'CLASSES FOR THE DEVELOPMENTAL OF FUNDAMENTAL MOVEMENTS SKILLS}

\begin{abstract}
This study investigated possible differences in the level of development of fundamental motor skills among preschool children aged between 4 and 7 years who regularly participate in sports (Artistic Gymnastics and Physical Education) and preschool children of the same age who participate only of Physical Education (PE), including comparison between ages. Twelve children who had two weekly PE classes formed the Physics Education Group (GEF) and twelve other children who had two weekly PE classes formed the group GA. For the evaluation of fundamental movement patterns, we used the


Instrumental Assessment of Fundamental Movements model. Data were evaluated by component as a checklist in which each movement pattern can be classified into initial, elementary or mature pattern. The data were analyzed for distribution (percentage) at each level of development. The results showed that children of GA showed better results compared to the GEF, except for the fundamental skills of manipulation. Thus, we conclude that the GA lessons contributed to better motor skills development locomotion and stabilization.

Key-Words: Fundamental skills; Artistic gymnastics; Physical Education

\section{APORTES DE LA CLASE DE GIMNASIA ARTÍSTICA PARA EL DESARROLLO DE LAS HABILIDADES BÁSICAS DE MOVIMIENTO}

\section{RESUMEN}

Este estudio investigó las posibles diferencias en los movimientos fundamentales entre los niños en edad preescolar que participan regularmente en la práctica de los deportes y en edad preescolar que participan sólo de La Educación Física, incluyendo La comparación de las edades. Doce niños que tenían dos clases de educación física semanales formaron el grupo de Educación Física (EF) y otros doce que tenían dos clases de educación física semanales y dos clases de gimnasia artística, el grupo (GA). Para la evaluación de los patrones fundamentales de movimiento, se utilizo el modelo de Evaluación Instrumental de Movimientos Fundamentales. Los datos fueron evaluados por componente como una lista en la que cada patrón de movimiento se puede clasificar en padrón inicial, primaria o madura. Se analizaron los datos para La distribución (porcentaje) en cada nivel de desarrollo. Los resultados indicaron que los niños de GA mostraron mejores resultados en relación AL grupo EF, excepto en los movimientos de manipulación. Por lo tanto, se concluye que las clases GA aliados de las clases de EFcontribuyeron a un mejor desarrollo motor en relación con los niños que eran simplemente Del grupo de Educación Física.

Palabra-Clave: Habilidades fundamentales; Gimnasia artística; Educación Física. 


\section{INTRODUÇÃO}

A infância é o período em que ocorrem as mudanças significativas em termos de desenvolvimento, sendo que estas alterações são determinadas por uma série de fatores relacionados tanto com o processo maturacional, quanto com as influências ambientais. No que se refere à sequência de desenvolvimento motor, as habilidades motoras fundamentais são decorrência da fase de movimentos rudimentares do período neonatal e representam um período no qual as crianças estão ativamente envolvidas na exploração e na experimentação das capacidades motoras de seus corpos. ${ }^{1}$ Este é um período para descobrir como desempenhar uma variedade de habilidades estabilizadoras, locomotoras e manipulativas, primeiras isoladamente e, depois combinadas. As crianças que estão desenvolvendo as habilidades fundamentais estão aprendendo a responder com controle motor e competência motora a vários estímulos. ${ }^{2}$

Durante a infância, a competência motora é considerada a capacidade de executar as habilidades motoras fundamentais com proficiência ${ }^{3}$ e constitui a base para a futura participação em atividades físicas que exigem movimentos especializados. ${ }^{4} \mathrm{Se}$ as habilidades motoras fundamentais não são devidamente desenvolvidas, uma criança pode enfrentar uma barreira de proficiência que pode limitar o seu desempenho e a capacidade de aprender novos movimentos e, portanto, dificultando a adesão em atividades físicas e esportes. ${ }^{4}$ Inclusive, estas são mais propensas a adotar um comportamento sedentário ao longo vida. ${ }^{5}$

A aquisição e o refinamento de habilidades motoras são influenciados por muitos fatores, sendo que estes fatores podem ser denominados de restrições. ${ }^{6}$ De acordo com o modelo proposto por Newell, ${ }^{6}$ a proficiência motora de crianças é influenciada por restrições relacionadas com a tarefa, o indivíduo e o contexto. Neste sentido, cada contexto esportivo tem exigências particulares e requer características e habilidades específicas, que delimitam o comportamento motor de uma criança. Adicionalmente, as demandas do ambiente tendem a delinear o repertório motor e agir sobre o nível de desempenho de cada habilidade motora, pois a partir das restrições que novos padrões coordenativos emergem. ${ }^{6}$ Com esses preceitos teóricos, Krebs, ${ }^{7}$ com uma perspectiva mais ecológica, também 
afirmou que múltiplos contextos em que uma criança está envolvida, de alguma forma, podem interferir com o processo de seu desenvolvimento motor.

Como parte do contexto, a influência ambiental no processo de desenvolvimento, tais como programas de atividade física e aulas de Educação Física têm sido demonstrada por diversos estudiosos ${ }^{8-10}$ e reforçam a necessidade de compreender como as diferentes restrições do ambiente interagem com a criança em desenvolvimento. Estas diferentes restrições que influenciam no desenvolvimento de padrões coordenativos, o que inclui as habilidades fundamentais, podem ser representadas pela prática de modalidades esportivas, pois as diferentes demandas representam diferentes restrições.

Newell ${ }^{6}$ acrescenta que cada contexto esportivo possui habilidades motoras específicas que foram sendo construídas e modificadas ao longo do tempo. Um exemplo disto é a modalidade de Ginastica Artística (GA), uma das modalidades consideradas mais antigas. Apesar da popularidade midiática da GA ter aumentado consideravelmente em nosso país na última década, frente aos resultados expressivos de ginastas brasileiros, como a medalha olímpica nas argolas de Arthur Zanetti nos Jogos Olímpicos de Londres (2012), o aumento de adeptos na modalidade no Brasil não seguiu o mesmo ritmo. ${ }^{11}$ Talvez, mas não somente, os preconceitos $^{12}$ referentes à modalidade ainda prevaleçam sobre os seus benefícios.

A GA é organizada com base nas habilidades básicas de caminhar, correr, saltar, equilibrar, rolar, balançar, girar, dentre outras. Quando realizados de forma planejada, sistematizada e sequenciada, as atividades desenvolvidas possuem potencial para intensificar os benefícios que esta prática esportiva pode propiciar. ${ }^{13}$ Por exigir diferentes habilidades motoras e condicionamento físico, a GA estimula a eficiência fisiológica, a força, flexibilidade, resistência e agilidade, estimula a criatividade, perseverança e coragem, ${ }^{14}$ ou seja, o desenvolvimento multilateral na criança. Desta maneira, a GA e seus equipamentos característicos - trave, trampolins, paralelas - é um esporte rico quanto ao ambiente de aula, que é responsável pelo interesse inicial na atividade de crianças e jovens. ${ }^{15}$

A variedade de habilidades realizadas nos diferentes aparelhos e a combinação ilimitada de possibilidades corporais permite a aplicação de um grande número de exercícios físicos Conexões: revista da Faculdade de Educação Física da UNICAMP, Campinas, v. 13, n. 3, p. 65-84, jul./set. 2015. ISSN: 1983-9030 
diferentes por sua forma e coordenação, aumentando as possibilidades motoras de seus praticantes. Leguet ${ }^{13}$ os organiza em 12 famílias, dentre os quais: suspender-se, apoiar-se, mover-se em bipedia, aterrissar, saltar, equilibrar-se, girar sobre si mesmo e acrescenta que são fundamentais não apenas para a GA, mas como base motora para diversas atividades esportivas. A partir destas propostas é possível assumir que aulas de GA auxiliam no desenvolvimento de habilidades fundamentais, mas ainda não foram encontrados estudos que a testaram.

Levando em consideração a importância do contexto no processo de desenvolvimento, surgiu a nossa curiosidade: A prática da Ginástica Artística pode auxiliar no desenvolvimento de habilidades motoras fundamentais de pré-escolares? Em caso positivo, há alguma classe de habilidades mais beneficiada? Desta forma, o objetivo deste estudo foi investigar possíveis diferenças nas habilidades fundamentais entre pré-escolares com idade entre quatro e sete anos que participam regularmente da prática de esportes (escolinha de Ginástica Artística) e pré-escolares da mesma idade que participam apenas da Educação Física escolar, e também verificar se existe alguma diferença entre as faixas etárias.

\section{MÉTODO}

\section{Amostra}

Participaram do estudo 24 crianças, todas do sexo feminino, com idade entre quatro e sete anos, de uma cidade interiorana do Brasil (TABELA 1). Os responsáveis pelos alunos assinaram um termo de consentimento antes do início da coleta de dados autorizando a participação das crianças no estudo. Este estudo foi aprovado pelo Comitê de Ética da Universidade Federal do Triângulo Mineiro (CEP/UFTM) sob o número 1705.

\section{Instrumentos e procedimentos}

Para a avaliação dos padrões fundamentais de movimento, foi utilizado o Modelo de Avaliação Instrumental dos Movimentos Fundamentais. ${ }^{16}$ Utilizou-se uma câmera filmadora digital (2.5 zoom, 3.2 megapixel, marca Sony-Handyean Vision), com tripé para registrar os movimentos. Após a filmagem, os padrões fundamentais de movimentos foram analisados e classificados por componentes. As crianças foram divididas em dois grupos sendo que as pertencentes ao grupo de GA realizavam a modalidade no projeto de extensão 
do Centro Universitário da Cidade. O nível de atividade física dos voluntários foi controlado sendo que para o grupo de GA as crianças praticavam a modalidade duas vezes por semana, com duração de uma hora, além da Educação Física escolar; e as crianças do grupo EF escolar realizavam apenas a Educação Física escolar em uma frequência de também duas vezes na semana, com duração de 50 minutos. Os conteúdos desenvolvidos nas aulas de EF foram os seguintes: circuitos de habilidades com o objetivo de trabalhar saltos, corridas, equilíbrio e giros; esportes, como mini voleibol, futsal e basquete, tendo como propósito a iniciação esportiva nos esportes e jogos como carimbada, bandeirinha, pique-pega, entre outros. Os materiais utilizados nas aulas basicamente foram bolas, cordas, arcos, giz e bancos. Esta informação foi adquirida no plano de ensino e plano de aula do professor de Educação Física responsável pelas aulas. Além das informações do plano de ensino também foi perguntado ao professor de Educação Física a forma na qual eram ministrados estes conteúdos. Conforme nos foi relatado, as atividades eram desenvolvidas mensalmente, ou seja, um mês de circuitos variados, um mês para cada esporte e um mês para jogos variados.

Para participar do estudo as crianças do grupo de GA deveriam estar engajadas na atividade há pelo menos seis meses. Utilizamos o tempo de prática de seis meses, pautados em estudos específicos da modalidade. Kumakura ${ }^{17}$ considerou experientes as crianças que praticavam GA há, pelo menos um ano. Públio e Tani ${ }^{18}$ confirmaram que é preciso cerca de três meses de prática para a compreensão de novos elementos ginásticos. Sendo assim, optamos pelo tempo médio de seis meses, ou seja, aqueles que já adquiriram benefícios motores da GA, mas não necessariamente são considerados experts na modalidade.

Com relação aos testes, foram realizados da seguinte forma: para o movimento equilíbrio em um só pé sobre a trave baixa $(45 \mathrm{~cm})$, a criança foi instruída a equilibrar-se nesta posição durante 5 segundos; no caminhar sobre a trave, a instrução foi para caminhar de frente pelos 5 metros da mesma; no arremessar, a instrução foi para arremessar uma bola de tênis o mais distante possível, sem a utilização de saltos ou corrida prévia, no movimento de quicar a bola, a instrução foi para quicar uma bola de borracha marca penalty n.10 na posição parado durante cinco segundos. Para o movimento correr, a criança foi instruída a correr numa direção de uma extremidade a outra de uma quadra poliesportiva, buscando atingir sua velocidade máxima, e para o movimento de saltar, Conexões: revista da Faculdade de Educação Física da UNICAMP, Campinas, v. 13, n. 3, p. 65-84, jul./set. 2015. ISSN: $1983-9030$ 
solicitou-se que a criança tentasse atingir o ponto mais distante que conseguisse, partindo de uma posição onde seus pés estariam parados e paralelos. Cada participante realizou três tentativas de cada habilidade testada, as quais foram filmadas com a câmera posicionada à $45^{\circ}$ em relação ao executante.

\section{Delineamento}

Os dois grupos, GA e EF foram subdivididos em função da idade em GA1 e EF1, com idade de 4-5 anos, e em GA2 e EF2, com idade de 6-7 anos (TABELA 1). Foram avaliados seis movimentos fundamentais que são divididos em: estabilizadores (equilíbrio em um só pé sobre a trave baixa e caminhar sobre a trave), manipulativos (arremessar e quicar a bola) e os locomotores (correr e saltar). Esta avaliação possibilita classificar os indivíduos nos estágios de desenvolvimento inicial, elementar e maduro, primeiro usando uma abordagem de configurações corporal total, seguida por abordagem de análise segmentaria individual (TABELA 1).

Tabela 1 - Descrição da amostra

\begin{tabular}{llrrr}
\hline Nome & Descrição & N & Média idade & \multicolumn{2}{c}{ DP } \\
\hline GA 1 & Grupo Ginástica Artística 4 e 5 anos & 6 & 4,66 & 0,47 \\
GA 2 & Grupo Ginástica Artística 6 e 7 anos & 6 & 6,16 & 0,37 \\
Subtotal & & 12 & & \\
GEF 1 & Grupo Educação Física 4 e 5 anos & 6 & 4,5 & 0,5 \\
GEF2 & Grupo Educação Física 6 e 7 anos & 6 & 6,5 & 0,5 \\
Subtotal & & 12 & & \\
\hline TOTAL & & 23 & & \\
\hline
\end{tabular}

Os dados foram avaliados por componente conforme a lista de checagem, no qual cada padrão de movimento pode ser classificado em padrão inicial, elementar ou maduro (para maiores esclarecimentos verificar McClenaghan e Gallahue). ${ }^{16}$ Os dados foram analisados em relação à distribuição (porcentagem) em cada nível de desenvolvimento. Foi utilizado o Modelo de Avaliação Instrumental dos Movimentos Fundamentais por ser uma referência de apoio a profissionais durante a condução de programas de atividades motoras para crianças. No presente estudo, buscando esta proximidade com o professor de Educação Física, optou-se por este instrumento, visto que seus resultados seriam aqueles observados em situações reais de ensino-aprendizagem. 


\section{RESULTADOS}

O Quadro 1 apresenta os resultados descritivos para o grupo GA1. A análise dos testes mostrou que para o componente equilíbrio em um só pé, caminhar sobre a trave, correr e saltar todas as crianças de prática de GA se encontraram no estágio elementar. Para o quicar e arremessar, todas as crianças se encontraram no estágio inicial.

Quadro 1 - Resultados da análise do grupo GA1

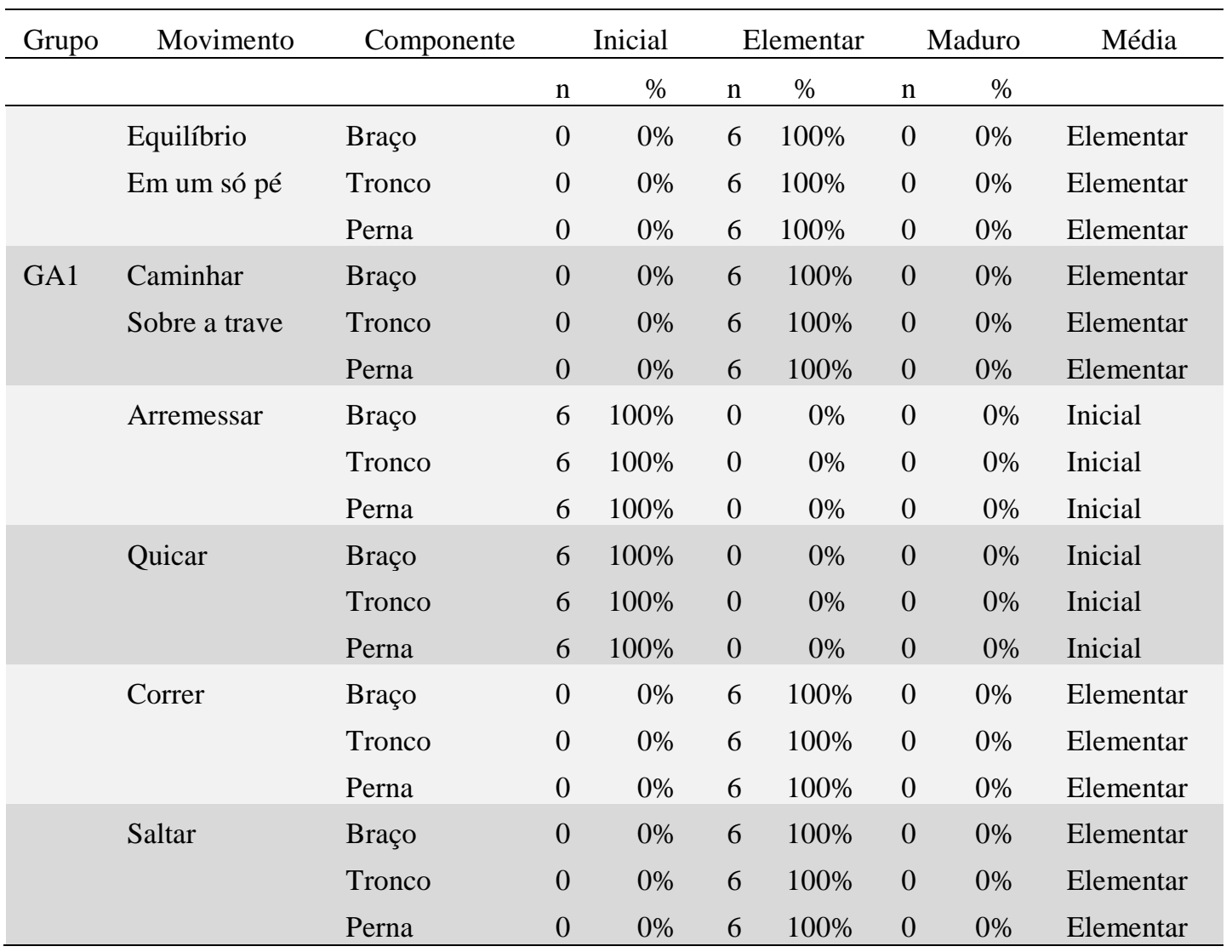

O Quadro 2 apresenta os resultados descritivos para o grupo GEF1. A análise dos testes mostrou que para todos os movimentos analisados (exceto correr) todas as crianças engajadas apenas na EF escolar se encontraram no estágio inicial. Para a habilidade de correr $50 \%$ das crianças encontraram-se no estágio inicial e 50\% no elementar. 
Quadro 2 - Resultados da análise do grupo GEF1

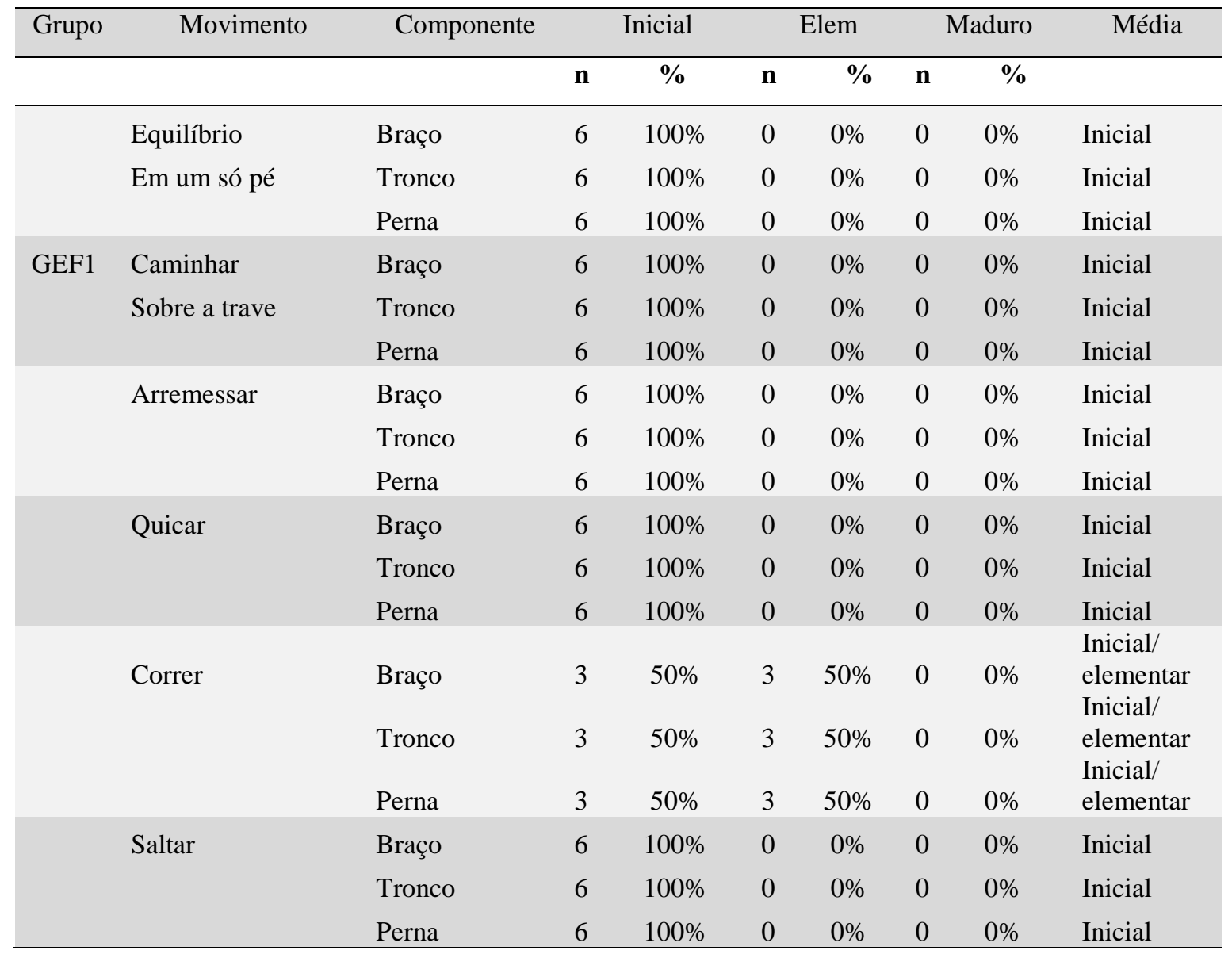

O Quadro 3 apresenta os resultados descritivos para o grupo GA2. A análise dos testes mostrou que para apenas o componente equilíbrio em um só pé, e caminhar sobre a trave, todas as crianças matriculadas na GA se encontraram no estágio maduro. Para o quicar e arremessar (componente de braço) as crianças se encontraram no estágio inicial e para correr e saltar no estágio elementar. 
Quadro 3 - Resultados da análise do grupo GA2

\begin{tabular}{|c|c|c|c|c|c|c|c|c|c|}
\hline \multirow[t]{2}{*}{ Grupo } & \multirow[t]{2}{*}{ Movimento } & \multirow[t]{2}{*}{ Componente } & \multicolumn{2}{|c|}{ Inicial } & \multicolumn{2}{|r|}{ Elem } & \multicolumn{2}{|c|}{ Maduro } & \multirow[t]{2}{*}{ Média } \\
\hline & & & n & $\%$ & n & $\%$ & $\mathbf{n}$ & $\%$ & \\
\hline & Equilíbrio & Braço & 0 & $0 \%$ & 0 & $0 \%$ & 6 & $100 \%$ & Maduro \\
\hline & Em um só pé & Tronco & 0 & $0 \%$ & 0 & $0 \%$ & 6 & $100 \%$ & Maduro \\
\hline & & Perna & 0 & $0 \%$ & 0 & $0 \%$ & 6 & $100 \%$ & Maduro \\
\hline \multirow[t]{3}{*}{ GA2 } & Caminhar & Braço & 0 & $0 \%$ & 0 & $0 \%$ & 6 & $100 \%$ & Maduro \\
\hline & Sobre a trave & Tronco & 0 & $0 \%$ & 0 & $0 \%$ & 6 & $100 \%$ & Maduro \\
\hline & & Perna & 0 & $0 \%$ & 0 & $0 \%$ & 6 & $100 \%$ & Maduro \\
\hline & \multirow[t]{3}{*}{ Arremessar } & Braço & 5 & $83 \%$ & 1 & $16 \%$ & 0 & $0 \%$ & Inicial \\
\hline & & Tronco & 0 & $0 \%$ & 6 & $100 \%$ & 0 & $0 \%$ & Elementar \\
\hline & & Perna & 0 & $0 \%$ & 6 & $100 \%$ & 0 & $0 \%$ & Elementar \\
\hline & \multirow[t]{3}{*}{ Quicar } & Braço & 4 & $66 \%$ & & $33 \%$ & 0 & $0 \%$ & Inicial \\
\hline & & Tronco & 0 & $0 \%$ & 6 & $100 \%$ & 0 & $0 \%$ & Elementar \\
\hline & & Perna & 0 & $0 \%$ & 6 & $100 \%$ & 0 & $0 \%$ & Elementar \\
\hline & \multirow[t]{3}{*}{ Correr } & Braço & 0 & $0 \%$ & 6 & $100 \%$ & 0 & $0 \%$ & Elementar \\
\hline & & Tronco & 0 & $0 \%$ & 6 & $100 \%$ & 0 & $0 \%$ & Elementar \\
\hline & & Perna & 0 & $0 \%$ & 6 & $100 \%$ & 0 & $0 \%$ & Elementar \\
\hline & \multirow[t]{3}{*}{ Saltar } & Braço & 0 & $0 \%$ & 6 & $100 \%$ & 0 & $0 \%$ & Elementar \\
\hline & & Tronco & 0 & $0 \%$ & 6 & $100 \%$ & 0 & $0 \%$ & Elementar \\
\hline & & Perna & 0 & $0 \%$ & 6 & $100 \%$ & 0 & $0 \%$ & Elementar \\
\hline
\end{tabular}

O Quadro 4 apresenta os resultados descritivos para o grupo GEF2. A análise dos testes mostrou que para o componente equilíbrio em um só pé e caminhar sobre a trave todas as crianças engajadas apenas na EF escolar se encontraram no estágio inicial. Para o arremessar, quicar, correr e saltar todas se encontraram no estágio elementar. 
Quadro 4 - Resultados da análise do grupo EF2

\begin{tabular}{|c|c|c|c|c|c|c|c|c|c|}
\hline \multirow[t]{2}{*}{ Grupo } & \multirow[t]{2}{*}{ Movimento } & \multirow[t]{2}{*}{ Componente } & \multicolumn{2}{|r|}{ Inicial } & \multicolumn{2}{|r|}{ Elem } & \multicolumn{2}{|c|}{ Maduro } & \multirow[t]{2}{*}{ Média } \\
\hline & & & n & $\%$ & $\mathbf{n}$ & $\%$ & n & $\%$ & \\
\hline & Equilíbrio & Braço & 6 & $100 \%$ & 0 & $0 \%$ & 0 & $0 \%$ & Inicial \\
\hline & Em um só pé & Tronco & 6 & $100 \%$ & 0 & $0 \%$ & 0 & $0 \%$ & Inicial \\
\hline & & Perna & 6 & $100 \%$ & 0 & $0 \%$ & 0 & $0 \%$ & Inicial \\
\hline \multirow[t]{3}{*}{ GEF2 } & Caminhar & Braço & 6 & $100 \%$ & 0 & $0 \%$ & 0 & $0 \%$ & Inicial \\
\hline & Sobre a trave & Tronco & 6 & $100 \%$ & 0 & $0 \%$ & 0 & $0 \%$ & Inicial \\
\hline & & Perna & 6 & $100 \%$ & 0 & $0 \%$ & 0 & $0 \%$ & Inicial \\
\hline & Arremessar & Braço & 0 & $0 \%$ & 6 & $100 \%$ & 0 & $0 \%$ & Elementar \\
\hline & & Tronco & 0 & $0 \%$ & 6 & $100 \%$ & 0 & $0 \%$ & Elementar \\
\hline & & Perna & 0 & $0 \%$ & 6 & $100 \%$ & 0 & $0 \%$ & Elementar \\
\hline & Quicar & Braço & 0 & $0 \%$ & 6 & $100 \%$ & 0 & $0 \%$ & Elementar \\
\hline & & Tronco & 0 & $0 \%$ & 6 & $100 \%$ & 0 & $0 \%$ & Elementar \\
\hline & & Perna & 0 & $0 \%$ & 6 & $100 \%$ & 0 & $0 \%$ & Elementar \\
\hline \multirow{3}{*}{\multicolumn{2}{|c|}{ Correr }} & Braço & 0 & $0 \%$ & 6 & $100 \%$ & 0 & $0 \%$ & Elementar \\
\hline & & Tronco & 0 & $0 \%$ & 6 & $100 \%$ & 0 & $0 \%$ & Elementar \\
\hline & & Perna & 0 & $0 \%$ & 6 & $100 \%$ & 0 & $0 \%$ & Elementar \\
\hline \multirow{2}{*}{\multicolumn{2}{|c|}{ Saltar }} & Braço & 0 & $0 \%$ & 6 & $100 \%$ & 0 & $0 \%$ & Elementar \\
\hline & & $\begin{array}{l}\text { Tronco } \\
\text { Perna }\end{array}$ & $\begin{array}{l}0 \\
0\end{array}$ & $\begin{array}{l}0 \% \\
0 \%\end{array}$ & $\begin{array}{l}6 \\
6\end{array}$ & $\begin{array}{l}100 \% \\
100 \%\end{array}$ & $\begin{array}{l}0 \\
0\end{array}$ & $\begin{array}{l}0 \% \\
0 \%\end{array}$ & $\begin{array}{l}\text { Elementar } \\
\text { Elementar }\end{array}$ \\
\hline
\end{tabular}

\section{DISCUSSÃO}

O objetivo do presente estudo foi verificar possíveis diferenças nas habilidades fundamentais entre pré-escolares com idade entre 4 e 7 anos que participam regularmente da prática de esportes (escolinha de GA) e pré-escolares da mesma idade que não participam, e seus efeitos foram comparados em duas faixas etárias. Os principais resultados mostraram que a modalidade de GA (para a idade de 4 e 5 anos) foi capaz de proporcionar um padrão de movimento compatível com a literatura ${ }^{16}$ em relação aos movimentos de estabilização e locomoção, e para a idade de 6 e 7 anos apenas em relação aos movimentos de estabilização. Para o grupo que realizava apenas a EF escolar, o padrão de movimento para a maioria dos movimentos fundamentais encontrava-se no estágio abaixo do recomendado.

Nossos resultados corroboram com os de Kumakura, ${ }^{17}$ que também encontrou influência positiva da Ginástica Artística no desenvolvimento motor em escolares. No estudo desta autora, foram analisadas 119 crianças entre 7 a 10 anos de idade, das quais 69 praticantes 
de atividades escolares e GA e 50 praticantes apenas de atividades escolares. Apesar de avaliarem o quociente motor e não o nível de desenvolvimento das habilidades fundamentais, os resultados demonstraram que a prática da GA teve melhor desempenho que o grupo de EF e que este não sofreu influência significativa dos níveis maturacionais nos grupos analisados.

Parece que as aulas de GA aliada as de EF propiciaram o desenvolvimento de habilidades básicas e consequentemente um desenvolvimento motor mais adequado. Os resultados observados indicam a importância da prática sistemática de atividades motoras para o melhor desenvolvimento das habilidades motoras fundamentais. Segundo Gallahue e Donnelly, ${ }^{1}$ à medida que as crianças aproximam-se de dois anos de idade, alterações acentuadas podem ser observadas na maneira como elas relacionam-se com o que as cerca. Nesta idade, elas já dominaram as habilidades motoras rudimentares que formam a base sobre a qual cada criança desenvolve ou refina os padrões motores fundamentais do início da infância. A fase de movimentos fundamentais se estende até aproximadamente sete anos de idade, quando as crianças passam a apresentar padrões de movimento mais consistentes e maduros. Porém, os resultados do presente estudo não corroboram com essa afirmação, pois apenas as crianças engajadas na GA foram capazes de apresentar este comportamento.

Schiavon et al. ${ }^{11}$ destacam que na modalidade de GA, os giros ao redor dos eixos longitudinal, transversal e anteroposterior exigem uma percepção do sentido de equilíbrio. Além disso, incluem a questão do sucesso da ginástica advir dos elementos acrobáticos, que exigem, desde os estágios iniciais da aprendizagem, a coordenação de padrões fundamentais de movimento, como o correr e saltar, o que pode explicar o melhor desempenho do grupo GA nas habilidades locomotoras. Além disso, o correr e o saltar exigem alta capacidade de aterrissagem no solo (estabilização do movimento). Estas informações reforçam a superioridade do grupo GA em relação ao GEF principalmente nos movimentos estabilizadores.

Voltando aos nossos resultados, para o grupo GA1 (4 e 5 anos) observamos que para os movimentos estabilizadores e locomotores as crianças alcançaram padrões compatíveis para a idade; e para o grupo GA2 (6 e 7 anos) as crianças alcançaram padrões compatíveis com a idade apenas para os movimentos estabilizadores. Ferraz ${ }^{19}$ analisou o padrão da Conexões: revista da Faculdade de Educação Física da UNICAMP, Campinas, v. 13, n. 3, p. 65-84, jul./set. 2015. ISSN: $1983-9030$ 
corrida em crianças de 4 a 9 anos de idade em que prevaleceu níveis rudimentares de ação. Surdi e $\mathrm{Krebs}^{20}$ verificaram crianças de seis anos que estavam no estágio elementar nos movimentos andar sobre a trave, saltar na horizontal, arremessar, correr, chutar e quicar. Uma das hipóteses para os baixos resultados volta-se para o que Carniel e Toigo ${ }^{21}$ elencam como pouca "aprendizagem ativa" na aula, ou seja, o tempo em que os alunos estão efetivamente engajados na prática tem se apresentado relativamente baixo.

Cotrim et al. ${ }^{9}$ e Lemos, Avigo e Barela ${ }^{10}$ acrescentam que as informações, demonstrações adequadas, feedback constante e possível carência de diversidade na prática podem acarretar em prejuízos motores.

Um fator que pode auxiliar as crianças a atingirem o estágio adequado nas habilidades fundamentais diz respeito ao envolvimento com habilidades esportivas. Essa questão foi investigada por Maforte e Benda ${ }^{22}$ e Siqueira, ${ }^{23}$ que observaram melhores níveis de desenvolvimento de crianças que praticam iniciação esportiva além de aulas de Educação Física regulares quando comparadas com crianças que somente frequentam aulas de Educação Física regulares. Fonseca et al. ${ }^{18}$ encontraram que quanto maior o tempo de prática na modalidade, melhores os resultados da coordenação motora, um dos componentes do desempenho motor. Contudo, Rocha, Rocha e Bertolasce ${ }^{24}$ apontou superioridade no nível motor entre crianças que realizam aulas de Educação Física para com aquelas que não a praticam. Assim, quanto mais tempo de estímulo, provavelmente melhores são os resultados neste âmbito.

A constatação de que a prática extracurricular (neste caso a GA, aliada a Educação Física) pode estar relacionada à promoção de desenvolvimento específico pode ser atribuída ao fato de que o conteúdo destas aulas compõe a base motora predominantemente de habilidades estabilizadoras e locomotoras. Uma verificação desta afirmação vem dos nossos resultados sobre os movimentos manipuladores. As atividades de GA não envolvem o manuseio e a manipulação de objetos e implementos relacionados com as habilidades manipulativas avaliadas neste estudo. Portanto, a prática de GA não propiciou qualquer benefício adicional para o desenvolvimento de movimentos fundamentais manipulativos. Por outro lado, a execução de exercícios no qual o corpo geralmente encontra-se em 
posições incomuns, como vôos, rotações, suspensões, em superfícies e alturas diferenciadas é a característica marcante da modalidade.

Ainda com relação à habilidade de manipulação de objetos, a pesquisa de Azevedo $^{25}$ comparou crianças entre 7 a 10 anos de idade que participavam somente de programas de Educação Física e crianças que além desta, praticavam a Ginástica Rítmica (GR). Diferentemente do que encontramos em nosso estudo, o grupo de GR obteve maior índice nas habilidades manipulativas em relação ao outro grupo. Uma característica da GR é o manuseio de seus aparelhos de pequeno porte, quais sejam: maças, bola, arco, fita e corda, o que pode levar ao melhor desenvolvimento desta classe de habilidades. Não obstante, o trabalho de Valentini ${ }^{26}$ encontrou que, mesmo em atividades esportivas múltiplas, entre crianças de 5 a 10 anos de idade, o desempenho relacionado à manipulação encontrou-se pobre ou inferior a média defendida por teóricos.

Apesar de Wrotniak ${ }^{27}$ e Fisher et al. ${ }^{28}$ averiguarem relação positiva entre a proficiência motora e o tempo despendido para as atividades físicas, o segundo autor constatou que este fato só não teve relação para a habilidade de manipulação. Estes dados em conjunto com os encontrados no presente estudo indicam que, para além de prática, a qualidade desta precisa ser repensada e em especial em relação aos movimentos manipulativos.

Araújo et al. ${ }^{29}$ chamam a atenção para um aspecto que necessita ser discutido acerca da importância de atividades regulares ao longo da infância, com conteúdo específico visando o desenvolvimento das habilidades motoras fundamentais no contexto escolar. Concordamos com estes autores e podemos ratificar esta informação ao observar nossos resultados, ou seja, observamos claramente que crianças envolvidas em atividades regulares além da Educação Física escolar têm capacidade de demonstrar proficiência motora no desempenho de habilidades motoras fundamentais, desde que tenham vivenciado experiências e atividades regulares voltadas para o desenvolvimento dessas habilidades motoras.

No entanto, não são todas as crianças que têm oportunidade de vivenciar tais experiências fora do contexto escolar. Portanto, a inclusão de aulas regulares de Educação Física no ensino fundamental que propiciem oportunidades de vivências motoras, mesmo em Conexões: revista da Faculdade de Educação Física da UNICAMP, Campinas, v. 13, n. 3, p. 65-84, jul./set. 2015. ISSN: 1983-9030 
conjunto com outras atividades, é primordial para o desenvolvimento esperado das habilidades motoras fundamentais. ${ }^{9}$ Neste processo, deve-se selecionar e propor um conteúdo adequado às características, necessidades, expectativas e interesses dos alunos; apropriado no sentido de estar um pouco além das capacidades e competências já adquiridas e ser suficientemente desafiador para despertar a motivação e o entusiasmo para a aprendizagem.

Quando falamos em desenvolvimento motor têm-se, basicamente, dois processos fundamentais: aumento de diversidade e de complexidade do comportamento. ${ }^{2} \mathrm{O}$ primeiro refere-se ao aumento na quantidade de elementos do comportamento e o segundo ao aumento da interação entre os elementos do comportamento. Por exemplo, a criança adquire o andar, e com base nesse padrão de movimento desenvolve o andar diversificado variando-o quanto aos parâmetros força, velocidade e direção. ${ }^{2} \mathrm{O}$ aumento de diversidade do comportamento resulta na ampliação do repertório motor na horizontalidade. Posteriormente, elementos do comportamento como andar, correr, saltar e arremessar interagem entre si para formar estruturas cada vez mais complexas de movimento, resultando na ampliação do repertório motor na verticalidade. Por exemplo, o elemento correr interage com o elemento quicar uma bola dando origem a uma estrutura mais complexa denominada de driblar. ${ }^{2}$

Utilizando estes exemplos para o presente estudo, parece que as crianças que fazem a Educação Física escolar aliada à GA mostraram uma maior diversidade de movimento, refletida no estágio mais avançado de desenvolvimento. Perotti e Pelegrini ${ }^{30}$ evidenciam o girar sobre si mesmo. Para os autores, o rolamento é executável para a maioria das crianças sem grande dificuldade, no entanto, a evolução e o desempenho do movimento mudam no decorrer dos anos e estímulos. Na GA, é possível diversificar suas possibilidades: podemos realizar o giro para frente, para trás, em suspensão, em voo, na posição grupada, estendida, carpada, dentre outros. ${ }^{13} \mathrm{O}$ melhor nível de desenvolvimento pode facilitar a posterior combinação destas mesmas habilidades fundamentais.

O envolvimento em atividade ou programas de atividade física tem sido apontado como uma das formas de pelo menos minimizar os efeitos deletérios da sociedade moderna, tais como estresse e obesidade. ${ }^{31}$ Portanto, as crianças do presente estudo que ganharam Conexões: revista da Faculdade de Educação Física da UNICAMP, Campinas, v. 13, n. 3, p. 65-84, jul./set. 2015. ISSN: $1983-9030$ 
competência, de acordo com o esperado, na realização das habilidades motoras fundamentais, tem condições de manter o envolvimento em atividades futuras, evitando, assim, o fenômeno denominado de barreira de proficiência motora, ${ }^{4}$ que pode ser um impeditivo de envolvimento em tais atividades futuras e se manterem como pessoas fisicamente ativas.

Talvez esforços para a melhoria do desempenho motor e elevar as competências esportivas na infância auxiliam a mudança no quadro do baixo envolvimento nas atividades físicas entre crianças e adolescentes no Brasil e no mundo, ${ }^{32}$ afinal, há uma associação positiva entre o nível de proficiência motora e o envolvimento em atividade física. A formação motora básica de qualidade, com desenvolvimento de movimentos fundamentais e notável repertório motor a uma maior quantidade de crianças, pode massificar a prática esportiva e até potencializar a formação de atletas no futuro.

Com base nos resultados, podemos concluir que as aulas de GA aliadas as de Educação Física escolar contribuíram para uma melhor competência motora quando comparada apenas a Educação Física escolar. Esta conclusão é embasada no fato de que os conteúdos ministrados nas aulas de EF tinham objetivos semelhantes aos conteúdos das aulas de GA, mas apenas quando as atividades da EF foram aliadas as de GA é que foram observadas melhorias na competência. No entanto, ainda assim, a GA não garantiu melhoria em todas as classes de habilidades motoras, apontando a necessidade de diversificar as atividades motoras nesta faixa etária e assim obter melhor alcance da competência motora da criança.

\section{REFERÊNCIAS}

${ }^{1}$ GALlaHUE, D. L.; DONNELLY, F. C. Educação Física desenvolvimentista para todas as crianças. 4. ed. São Paulo, Phorte, 2008.

${ }^{2}$ TANI, G.; BASSO, L.; CORRÊA, U. C. O ensino do esporte para crianças e jovens: considerações sobre uma fase do processo de desenvolvimento motor esquecida. Revista Brasileira de Educação Física e Esporte, São Paulo, v. 26, n. 2, p. 339-50, abr. 2012. 
${ }^{3}$ STODDEN, D. F. et al. A developmental perspective on the role of motor skill competence in physical activity: an emergent relationship. Quest, Chicago, v. 60, n.1, p. 290-306, jan. 2008.

${ }^{4}$ CLARK, J. E. On the problem of motor skill development. Journal of Physical Education, Recreation and Dance, Reston, v. 78, n. 2, p. 39-45, jun. 2007.

${ }^{5}$ HARDY, L. et al. Fundamental movement skills among Australian preschool children. Journal of Science and Medicine in Sport/Sports Medicine, Austrália, v. 13, n. 3, p. 503-508, nov. 2010.

${ }^{6}$ NEWELL, K. M. Constraints on the development of coordination. In: WADE, M. G.; WHITING, H. T. A. (Org). Motor development in children: aspects of coordination and control. Boston: Martin Nijhoff, 1986.

${ }^{7}$ KREBS, R. J. A teoria dos sistemas ecológicos: um paradigma para a educação infantil. Santa Maria: Ed. da UFSM, 1997.

${ }^{8}$ BASSO, L.; MARQUES, I.; MANOEL, E. J. Colletive behavior of componentes in overarm throwing patern. Journal of Movement Studies, Edinburgh, v. 48, n. 2, p. 1-14, jan. 2005.

${ }^{9}$ COTRIM, J. R et al. Desenvolvimento de habilidades motoras fundamentais em crianças com diferentes contextos escolares. Revista de Educação Física/UEM, Maringá, v. 22, n. 2, p. 523-533, ago. 2011.

${ }^{10}$ LEMOS, A. G.; AVIGO, E. L.; BARELA, J. A. Physical Education in kindergarten promotes fundamental motor skill development. Advances in Physical Education, Boston, v. 2, n.1, p. 17-21, sept. 2012.

${ }^{11}$ SCHIAVON, L. M. et al. Panorama da ginástica artística brasileira de alto rendimento esportivo: progressão, realidade e necessidades. Revista Brasileira de Educação Física e Esporte, São Paulo, v. 27, n.1, p. 423-436, jan. 2013. Conexões: revista da Faculdade de Educação Física da UNICAMP, Campinas, v. 13, n. 3, p. 65-84, jul./set. 2015. ISSN: $1983-9030$ 
${ }^{12}$ TSUKAMOTO, M. H. C.; KNIJNIK, J. D. Ginástica artística e representações de masculinidades no Brasil. Revista Mackenzie de Educação Física e Esporte, São Paulo, v. 7 , n. 2, p. 111-118, abr. 2008.

${ }^{13}$ LEGUET, J. As ações motoras em ginástica esportiva. São Paulo: Manole, 1987.

${ }^{14}$ WERNER, P. H. From teaching children gymnastic: becoming a master teacher. Champaing: Human Kinetics, 1994.

${ }^{15}$ CARBINATTO, M. V. et al. Motivação e ginástica artística no contexto extracurricular. Conexões, Campinas, v. 8, n.1, p. 124-145, abr. 2010.

${ }^{16}$ MCClenaghan, B. A.; GALlahuE, D. L. Movimientos fundamentales: su desarrollo y rehabilitación. Buenos Aires: Editorial Medica Panamericana, 1985.

${ }^{17}$ KUMAKURA, RS. Influência da prática de ginástica artística no desenvolvimento motor de escolares. 2012. 96f. Dissertação (Mestrado em Ciências da Saúde) Universidade Federal de Sergipe, Sergipe, 2012.

${ }^{18}$ PUBLIO, N. S.; TANI, G. Aprendizagem de habilidades motoras seriadas da ginástica olímpica. Revista Paulista de Educação Física, São Paulo, v. 7, n. 1, p. 58-68, nov. 1993.

${ }^{19}$ FERRAZ, O. L. Desenvolvimento do padrão fundamental de movimento correr em crianças: um estudo semi-longitudinal. Revista Paulista de Educação Física, São Paulo, v. 6, n. 2, p. 26-34, jul. 1992.

${ }^{20}$ SURDI, A. C.; KREBS, R. J. Estudo dos padrões fundamentais de movimento de préescolares que participam de um programa de desenvolvimento infantil do SESI da cidade de Videira/SC. Kinesis, Santa Maria, v. 6, n. 21, p. 57-69, nov. 1999.

${ }^{21}$ CARNIEL, M. Z.; TOIGO, A. M. O tempo de aprendizagem ativo nas aulas de Educação Física em cinco escolas particulares de Porto Alegre, RS. Associação 
Brasileira de Pesquisa em Educação em Ciências. 2006. Disponível em: <http://www.fc.unesp.br/abrapec/revistas/v3n3a2.pdf>. Acesso em: 4 dez. 2010.

${ }^{22}$ MAFORTE, J. P. G.; BENDA, R. N. Iniciação esportiva ao futsal: uma oportunidade para o desenvolvimento motor da criança. In: CONGRESSO CIENTÍFICO LATINOAMERICANO FIEP-UNIMEP, 2000, Piracicaba. Anais... Piracicaba: FIEP: UNIMEP, 2000. v 1.p. 553.

${ }^{23}$ SIQUEIRA, M. Efeitos de um programa de iniciação esportiva ao futsal sobre os padrões fundamentais de movimento em crianças de 7 e 8 anos. 2006. 120f. Trabalho de Conclusão de Curso (Especialização em Educação Física) - Escola de Educação Física, Fisioterapia e Terapia Ocupacional, Universidade Federal de Minas Gerais, Belo Horizonte, 2006.

${ }^{24}$ ROCHA, P. G. M.; ROCHA, D. J. O.; BERTOLASCE, A. L. A influência da iniciação ao treinamento esportivo sobre o desenvolvimento motor na infância: um estudo de caso. Revista da Educação Física/UEM, Maringá, v. 21, n. 4, p. 469-477, abr. 2010.

${ }^{25}$ AZEVEDO, K. A. A Influência de um programa de ginástica rítmica sobre o desenvolvimento motor e a percepção de competência de crianças. 2012. 50f. Monografia (Especialização em Educação Física) - Escola de Educação Física, Universidade Federal do Rio Grande do Sul, Porto Alegre, 2012.

${ }^{26}$ VALENTINI, N. C. Percepções de competência e desenvolvimento motor de meninos e meninas: um estudo transversal. Movimento, Porto Alegre, v. 8, n. 2, p. 51-62, ago. 2002.

${ }^{27}$ WROTNIAK, B. H. The relationship between motor proficiency and physical activity in children. Pediatrics, Elk Grove, v. 118, n.17, p. 1758-1765, jan. 2006.

${ }^{28}$ FISHER, A. et al. Fundamental movement skills and habitual physical activity in young children. Medicine and Science in Sports and Exercise. Hagerstown, v. 37, n. 5, p. 684688, sept. 2005. 
${ }^{29}$ ARAUJO, M. P. et al. Contribuição de diferentes conteúdos das aulas de Educação Física no ensino fundamental I para o desenvolvimento de habilidades motoras fundamentais. Revista Brasileira de Medicina do Esporte, São Paulo, v.18, n.1, p. 153-157, fev. 2012.

${ }^{30}$ PEROTTI, A.; PELLEGRINI A. M. Organização espaço temporal do rolamento para frente. Movimento, Porto Alegre, v. 2, n. 4, p. 9-20, out. 2001.

${ }^{31}$ NELSON, T. F. et al. Do youth sports prevent pediatric obesity? a systematic review and commentary. Current Sports Medicine Reports, Philadelphia, v. 10, n. 8, p. 360-370, apr. 2011.

${ }^{32}$ PEARCE, M. S. et al. Early predictors of objectively measured physical activity and sedentary behaviour in 8-10 year old children: the gateshead millennium study. Plos One, Cambridge, v. 7, n.14, p. 379-375, jan. 2012.

Recebido em: 16 mar. 2015 Aceito em: 31 jul. 2015 Contato: suzi.ps@ig.com.br 